\title{
The sixth international RASopathies symposium: Precision medicine-From promise to practice
}

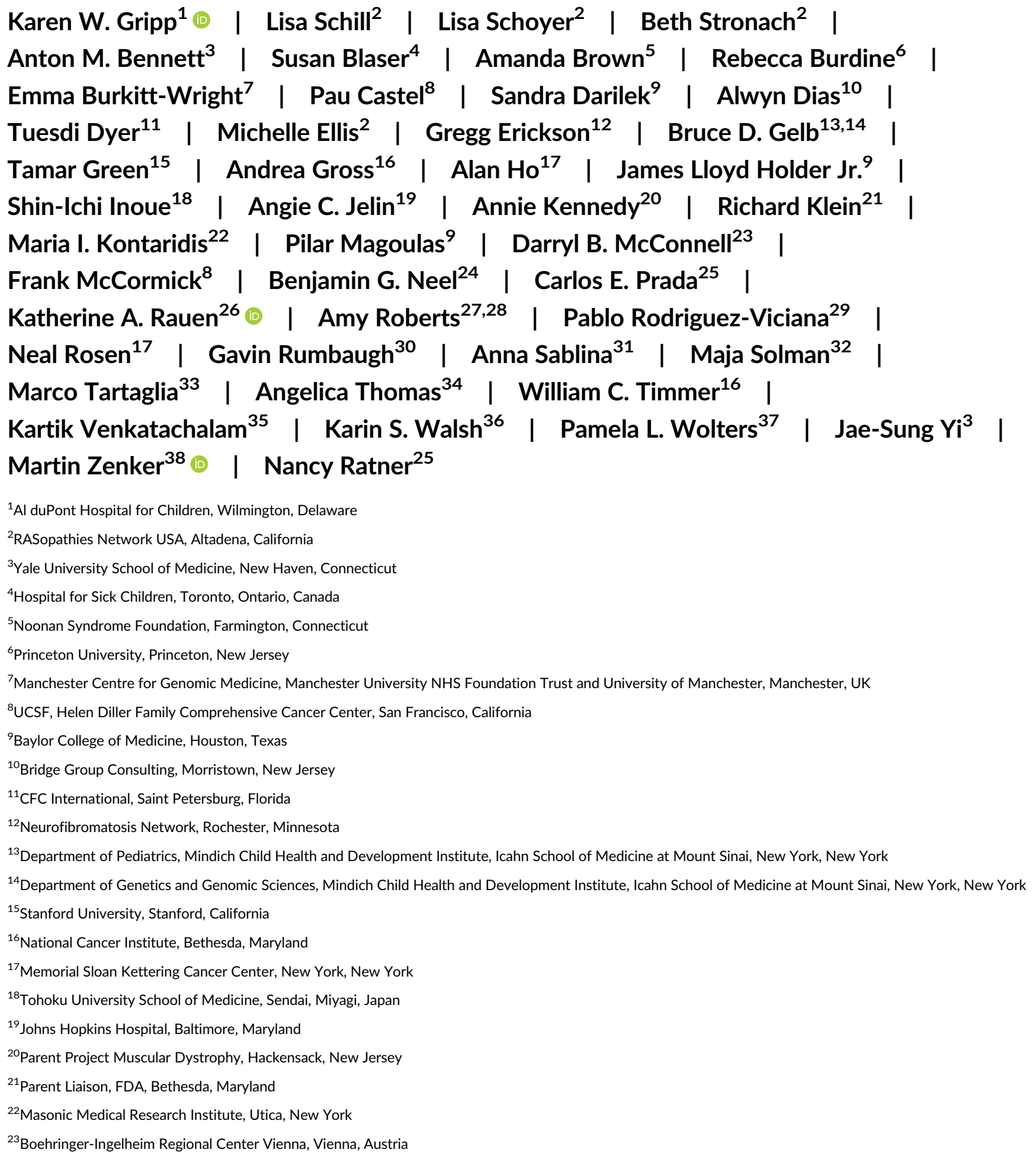




\footnotetext{
${ }^{24}$ Perlmutter Cancer Center and NYU School of Medicine, NYU Langone Health, New York, New York

${ }^{25}$ Cincinnati Children's Hospital Medical Center and University of Cincinnati, School of Medicine, Cincinnati, Ohio

${ }^{26}$ Department of Pediatrics, Division of Genomic Medicine, University of California Davis, Sacramento, California

${ }^{27}$ Department of Cardiology, Division of Genetics, Boston Children's Hospital, Boston, Massachusetts

${ }^{28}$ Department of Pediatrics, Division of Genetics, Boston Children's Hospital, Boston, Massachusetts

${ }^{29}$ UCL Cancer Institute, London, UK

${ }^{30}$ The Scripps Research Institute, Jupiter, Florida

${ }^{31}$ VIB-KU Leuven Center for Cancer Biology, Leuven, Belgium

${ }^{32}$ Hubrecht Institute-KNAW and University Medical Center Utrecht, Utrecht, The Netherlands

${ }^{33}$ Genetics and Rare Diseases Research Division, Ospedale Pediatrico Bambino Gesù-IRCCS, Rome, Italy

${ }^{34}$ Costello Syndrome Family Network, Woodinville, Washington

${ }^{35}$ McGovern Medical School, University of Texas Health Science Center, Houston, Texas

${ }^{36}$ Children's National Hospital \& The George Washington School of Medicine, Washington, District of Columbia

${ }^{37}$ Pediatric Oncology Branch, National Cancer Institute, Bethesda, Maryland

${ }^{38}$ University Hospital Magdeburg, Institute of Human Genetics, Magdeburg, Germany
}

\section{Correspondence}

Karen W. Gripp, Al duPont Hospital for

Children, Wilmington, DE.

Email: kgripp@nemours.org

Nancy Ratner, Cincinnati Children's Hospital Medical Center and University of Cincinnati, School of Medicine, Cincinnati, $\mathrm{OH}$.

Email: nancy.ratner@cchmc.org

\section{Funding information}

International Costello Syndrome Support Group; Children's Tumor Foundation; UAB School of Medicine; Prevention Genetics; Boehringer Ingelheim; KURA oncology; IGIA pharmaceuticals; National Cancer Institute; $\mathrm{NIH}$, Grant/Award Number: 1R13TR002780-01

\begin{abstract}
The RASopathies are a group of genetic disorders that result from germline pathogenic variants affecting RAS-mitogen activated protein kinase (MAPK) pathway genes. RASopathies share RAS/MAPK pathway dysregulation and share phenotypic manifestations affecting numerous organ systems, causing lifelong and at times life-limiting medical complications. RASopathies may benefit from precision medicine approaches. For this reason, the Sixth International RASopathies Symposium focused on exploring precision medicine. This meeting brought together basic science researchers, clinicians, clinician scientists, patient advocates, and representatives from pharmaceutical companies and the National Institutes of Health. Novel RASopathy genes, variants, and animal models were discussed in the context of medication trials and drug development. Attempts to define and measure meaningful endpoints for treatment trials were discussed, as was drug availability to patients after trial completion.
\end{abstract}

\section{KEYWORDS}

cardio-facio-cutaneous syndrome, Costello syndrome, kinases, neurofibromatosis, Noonan syndrome, RASopathy

\section{1 | INTRODUCTION}

The promise of precision medicine is based on the premise that understanding the molecular abnormalities driving medical conditions will enable targeted drug treatment on a patient-specific level. The hope for precision medicine is that treatment tailored to an individual's disease-causing genomic, metabolic, and pharmacogenomic variants will result in improved outcomes. The RASopathies, a group of conditions caused by germline pathogenic variants affecting the RAS/ERK mitogen activated protein kinase (RAS/MAPK) pathway, are well positioned for targeted treatment because they can have severe and lifethreatening manifestations. These may be congenital, and/or occur throughout the patients' life. Candidate drugs exist throughout the RAS/ERK pathway, given the major role of this signaling pathway in driving cancer. The Sixth International RASopathies Symposium, held August 2-4, 2019 in Baltimore, MA, aimed to further precision medicine approaches by highlighting the genetic causes of RASopathies, and their biological effects resulting in medical problems. Here, we share the proceedings of this meeting and invite interested parties to participate in this research effort.

\subsection{Novel pathogenic variants and novel genes}

Katherine Rauen opened the meeting by delineating the complexity of how RASopathies may be defined. RASopathies are phenotypically evolving multiple congenital anomaly syndromes that typically require a team of medical specialists throughout the patient's lifespan 
(Tidyman \& Rauen, 2016). RASopathy genes encode components of the Ras/ERK pathway and include several RAS proteins (HRAS, KRAS, NRAS, RRAS, RRAS2, RIT1), RAS exchange factors (SOS1, SOS2), GTPase activating proteins (NF1 and RASAL1), E3 ubiquitin ligases $(C B L)$, and downstream kinases functioning as signal transducers in the MAPK cascade (RAF and MAP2K genes). Session 1 , chaired by Marco Tartaglia, focused on additional genes recently implicated in RASopathies and newly characterized circuits controlling RAS-MAPK signaling. Phenotypic features associated with pathogenic variants in a subset of these genes were discussed. Finally, two talks were dedicated to the biology of SYNGAP1 and the disorder caused by SYNGAP1 haploinsufficiency.

Martin Zenker presented variant and clinical data from a large cohort of patients with Noonan syndrome (NS) collected in the NSEuroNet consortium. He focused on NS resulting from LZTR1 variants (OMIM \#616564 and \#605275). The data suggest that, aside from PTPN11, LZTR1 is among the most commonly mutated genes implicated in NS (similar in frequency to RAF1 and RIT1). The phenotypes associated with dominant or recessive LZTR1 variants fall within the NS clinical spectrum, and hypertrophic cardiomyopathy (HCM) is common. Sixty-five percent of those with LZTR1 variants have dominant NS. In the remaining cases, the mode of transmission is recessive or remains uncertain, as functional characterization of identified missense variants has not been performed, or because systematically scanning the noncoding portions of the gene may reveal additional variants. Notably, the distribution pattern of dominant and recessive LZTR1 variants is largely mutually exclusive. The former, all missense, cluster within the solvent exposed portions of the $\mathrm{N}$-terminal Kelch domains that mediate substrate binding, and the latter, including both missense and truncating variants, scatter throughout the entire coding region and adjacent intronic regions.

Anna Sablina found that complete loss of Lztr1 function is embryonic lethal in mice. Lztr1 haploinsufficiency recapitulates NS, with major features including reduced growth, craniofacial dysmorphia, and cardiac defects, including cardiac hypertrophy. She discussed LZTR1 biology and evidence establishing its role in controlling RAS signaling. LZTR1 encodes a member of the BTB/POZ protein superfamily that functions as an adaptor for the cullin 3 (CUL3) ubiquitin ligase complex, a multisubunit RING-class E3 ligase implicated in protein monoand poly-ubiquitination. Mass spectrometry studies revealed that LZTR1 and CUL3 form a complex implicated in RAS ubiquitination. Interaction studies indicate that multiple RAS subfamily members may be substrates of this complex. Notably, LZTR1-CUL3-mediated RAS ubiquitination does not promote RAS degradation; instead, it alters RAS subcellular localization by impairing RAS association with membranes. New data indicate that disease-associated LZTR1 variants can affect RAS ubiquitination by disrupting LZTR1-CUL3 complex formation or diminishing the LZTR1-CUL3 complex interaction with RAS proteins, resulting in defective RAS ubiquitination and enhanced RAS signaling.

Three abstracts submitted by junior investigators were selected for platform presentation. Pau Castel recently showed that RIT1 is also a target of LZTR1-mediated ubiquitination (Castel et al., 2019).
He reported on an Lztr1 knockout mouse model. Heterozygous mice were healthy and without NS features. Homozygous mice were embryonic lethal due to extensive hemorrhage between embryonic days E15.5 and 18.5. Lztr1 homozygous embryo hearts showed hypertrophic cardiac valves and sporadic septal defects. After back crossing to a different, more permissive, background (129Sv), the homozygous knockout mice survived and showed a typical NS phenotype including small size, craniofacial features, cardiomegaly, and splenomegaly suggesting background modifier effects. Like children with biallelic LZTR1 variants, the homozygous knockout mice had increased RIT1 levels. These mouse models can be used to study the pathogenesis of LZTR1-associated NS and to test pharmacological treatments.

Karen Gripp presented the clinical phenotype associated with de novo missense variants in PPP1CB, which encodes the protein phosphatase 1 catalytic subunit beta (PP1C). Major features include short stature, relative macrocephaly, distinctive facial features, congenital cardiac defects, pigmentary anomalies, and variable cognitive deficit, all common characteristics of NS and most other RASopathies. The subjects carrying PPP1CB pathogenic variants have a phenotype resembling Mazzanti syndrome, in which a recurrent activating variant in SHOC2 (p.Ser2Gly) causes a disease characterized by features resembling NS and distinctive ectodermal abnormalities, also called NS-like disorder (OMIM\# 617506). Affected individuals have loose anagen hair, slow growing hair with a defective root structure that is easily pulled from the scalp (OMIM\# 607721). Subjects with SHOC2 or PPP1CB variants show behavioral anomalies and cognitive defects, GH deficiency, and dark skin are additional common features (Gripp et al., 2016; Ma et al., 2016). No cancer has been reported in the 16 individuals with PPP1CB variants; the small number of cases does not yet allow genotype-phenotype correlations.

Pablo Rodriguez-Viciana presented the role of a PP1C, SHOC2, and MRAS complex in RAS-MAPK signaling. SHOC2 is a scaffold protein mediating RAS-promoted activation of the ERK MAPK cascade in response to extracellular stimuli. The SHOC2 protein consists almost entirely of leucine-rich repeats, and is a positive modulator of RASERK signaling because it mediates membrane translocation of the catalytic subunit(s) of protein 1 (PP1C). PP1C is required for RAF1 activation and the PP1C/SHOC2/MRAS complex specifically promotes RAF1 dephosphorylation at a conserved inhibitory serine residue (Ser259), which in turn enables protein dimerization and activation. RASopathy-associated SHOC2, MRAS, and PPP1CB mutants cause enhanced MEK phosphorylation by promoting an augmented or more stable binding of the three proteins forming the MRAS/SHOC2/ PPP1CB complex (Young et al., 2018). Of note, MRAS/SHOC2/PP1C complex-dependent and complex-independent mechanisms of RAF activation exist, which are thought to differentially modulate RAS-ERK signaling output in varying cellular contexts.

Frank McCormick analyzed the interaction between two RASopathy proteins, the NF1 gene product, Neurofibromin, and SPRED1. SPRED1 is necessary for localization of Neurofibromin to active RAS. The EVH1 domain of SPRED1 binds to the GAP domain of NF1, allowing interaction of neurofibromin with RAS-GTP. Phosphorylation of serine 105 of SPRED1, within the EVH1 domain, 
appears key to this process. RASopathy disease-associated variants cluster at this interface and abolish NF1-SPRED1 binding. NF1 exists as a high-affinity homodimer, in head-to-tail conformation, with unidentified contact points. Full-length NF1 is relatively inactive as a GAP for RAS, compared to NF1 fragments. The dimerization of NF1 suggests the potential for dominant negative effects of certain disease-associated variants, which may result in divergent mechanistic, and hence phenotypic, effects. Missense substitutions of NF1 codons 844-848, associated with particularly severe disease manifestations (Koczkowska et al., 2018), are candidates for these effects. The half-life of NF1 mutated at codon 848 appears significantly reduced in vitro.

Two talks focused on SYNGAP1 and the neurodevelopmental disorder (OMIM\# 612621; mental retardation, autosomal dominant 5) resulting from SYNGAP1 haploinsufficiency, with a particular focus on the clinical and functional overlap with RASopathies. Gavin Rumbaugh and Jimmy Holder outlined the clinical features of patients with pathogenic loss-of-function variants in SYNGAP1, which mainly include epilepsy, developmental delay, cognitive deficits, autism spectrum disorder, and other behavioral abnormalities. Individuals with this neurodevelopmental disorder have few symptoms not attributable to the central nervous system. SYNGAP1 encodes a protein that contains a RAS-GAP-related domain, which is highly expressed in brain and is characterized by alternative isoforms. This GAP largely localizes to dendritic spines in neocortical pyramidal neurons. It negatively controls RAS function and also regulates the activity of other small GTPases (e.g., RAP and RAB proteins). It is possible that the enhanced signal traffic through multiple GTPases of the RAS superfamily contribute to the clinical phenotype of patients with defective SYNGAP1 function. Further research assessing the significance of RAS signaling dysregulation associated with SYNGAP1 haploinsufficiency is required to define the SYNGAP1-related disorder as a RASopathy.

\section{2 | New functions of RASopathy genes}

Emma Burkitt-Wright moderated a session with Shin-ichi Inoue presenting on the metabolic phenotyping of a novel HRAS G12S mouse model of Costello syndrome (CS; OMIM\# 218040; Oba et al., 2018). Heterozygous animals have a phenotype reminiscent of human CS, with craniofacial features and cardiomyocyte hypertrophy, as seen in other mouse models of RASopathies (Araki et al., 2004; Schuhmacher et al., 2008; Urosevic et al., 2011). Less clearly related to human CS, but reminiscent of other HRAS-mutated mouse models (Schuhmacher et al., 2008), nephromegaly with fibrosis was identified. Mice fed a high fat diet were resistant to weight gain, but excess mortality was seen. Histological and biochemical investigation revealed microvesicular hepatosteatosis and impaired fatty acid oxidation: tandem mass spectrometry showed elevated blood acylcarnitines C16 and C18 (C18, C18.1, and C18.2) and lower fasting ketone levels than in wild-type animals. Decreased mitochondrial $\beta$-oxidation gene expression and lower blood glucose were demonstrated in mutant mice, after either 30-min fast or intraperitoneal glucose injection. These findings may have relevance to the hypoglycemia observed in patients with CS.

\section{3 | Therapeutic inhibitors: Preclinical studies and clinical trials}

Elegant work is ongoing to understand the dynamics of RAS-ERK signaling in RASopathies, and to block this pathway for therapeutic benefit, as envisioned in precision medicine. Targeting RAS itself or upstream and down-stream proteins in the RAS-MAPK cascade is being studied. Darryl McConnell discussed RAS as a potential drug target because RAS proteins play a central role in oncogenic signaling. Activated RAS promotes the formation of RAF homo- and hetero-dimers, which induce downstream signaling. An oncogene known since 1982, one in seven tumors is driven by KRAS, making it a promising cancer drug target. Unfortunately, there are no approved therapeutic agents against KRAS, because it lacks the easily targeted deep binding pockets present in kinases. Boehringer Ingelheim is pursuing multiple approaches to drug KRAS. Examples are the KRAS exchange factor (SOS1) inhibitor BI-3406 and the GTP-RAS inhibitor BI-2852, which exhibit nanomolar potency. Boehringer Ingelheim maintains an open innovation portal (openme.com) to allow access to these and other chemical probes for biological investigations. At present, these agents are available for nonclinical studies only. Recently, a clinical trial with the SOS1::KRAS protein-protein interaction inhibitor BI 1701963 alone and in combination with trametinib in patients with KRAS mutated solid tumors has been reported.

Neal Rosen's keynote lecture covered oncogenic RAF1 variants and differentially activated feedback loops that develop after RASERK activation. The different frequencies of pathogenic variants in the RAS-ERK pathway genes were evaluated in a set of sporadic cancers. RAF proteins can homo or heterodimerize. BRAF variants classified into three groups. Of 200 mutated BRAF alleles, V600E was by far the most frequent, and acts through a Class I, classically activating, mechanism. Other substitutions of codon 600 are also constitutively active, and like $\mathrm{V} 600 \mathrm{E}$ result in mutant BRAF proteins that signal as monomers. These Class I activating mutations result in profound feedback inhibition of RAS-GTP and are RAS-independent. A substitution involving the neighboring residue, $\mathrm{K} 601 \mathrm{E}$, is also activating, but this BRAF protein signals as a homodimer. Class II mutated BRAF proteins such as BRAF K601E function as activated, constitutive, RASindependent dimers. Many BRAF translocations identified in cancer result in deletion of an $\mathrm{N}$-terminal portion of the protein, which is replaced by the fusion partner. This deletion causes these fusions to function in a similar manner as the Class II mutants: as constitutive, RAS-independent activated dimers. By contrast, Class III BRAF mutants are hypoactive or kinase-dead and RAS-dependent. They bind more tightly to WT RAS than WT RAF and activate signaling by heterodimerizing with and activating WT CRAF when bound to RAS. Since activated ERK feedback inhibits RAS, these mutants often coexist with NF1 loss or other lesions that activate RAS, including many that also cause RASopathies. In some carcinomas, activation of WT 
receptor tyrosine kinases is sufficient to drive adequate RAS activation. The RAF inhibitors used clinically preferentially inhibit RAFmonomers (BRAF V600 alleles) and are generally ineffective in treating BRAF fusion and Class II mutant driven tumors. Currently, these tumors can only be treated with MEK inhibitors. Two types of RAF dimer inhibitors have been described. Pan-RAF inhibitors inhibit all tumors driven by BRAF mutant monomers, Class II BRAF mutants and fusions more potently than normal cells and RAS-driven tumors. A second class selectively disrupts BRAF containing homo and heterodimers, but spares CRAF homodimers (Yao et al., 2019). As predicted, these drugs inhibit Class I, II, and fusion driven tumors without obvious toxicity in patients.

Currently, RASopathies may be treated with MEK inhibitors, but these have a narrow therapeutic index due to cutaneous and other toxicities. MEK inhibitors are very effective and well tolerated in most patients with pediatric and other tumors containing only one or few mutant genes activating ERK-signaling. One example is patients with Langerhans' and non-Langerhans histiocytosis, in whom almost all tumors are driven by ERK signaling. Multiple MEK inhibitors with varying properties are in clinical trial or use. Trametinib is very potent, has a long half-life and causes significant toxicity. In contrast, selumetinib is a weaker drug with a short half-life, effective and welltolerated in neurofibromas. The toxicity of MEK inhibitors can be ameliorated by intermittent dosing and by combining them with RAF monomer inhibitors, which activate ERK signaling in normal cells. Strategies taking into account the complex dynamics of these signaling networks and the specific biochemical properties of the drugs will be necessary to explore effective therapies for conditions associated with aberrant Ras-ERK signaling.

PTPN11, the gene most commonly mutated in NS and Noonan syndrome with multiple lentigines (NSML), encodes Src homology region 2-containing protein tyrosine phosphatase 2 (SHP2), a nonreceptor phospho-tyrosine phosphatase. Preclinical and clinical data demonstrate that SHP2 promotes tumor progression in leukemia and solid tumors. Ben Neel presented work indicating that SHP2 blockade can overcome resistance to MEK inhibitors in many cancers. Compensatory reactivation of RAS is observed following MEK inhibition, and blocking SHP2 is a potential means to abolish this. For example, NF1-mutated malignant peripheral nerve sheath tumors (MPNST) are frequently fatal and single agent chemotherapy, even with pathway-focused MEK inhibitors, has not demonstrated dramatic effect, while dual blockade of RAS-ERK and PI3K/ AKT pathways is precluded by excessive toxicity. RTK overactivation has been observed in MPNST cells treated with the MEK inhibitor trametinib, resulting in reactivation of RAS and MEK proteins, preventing effective ERK inhibition. This reactivation is blocked by SHP099, an allosteric SHP2 inhibitor that prevents RASexchange, thereby decreasing RAS-GTP loading for wild-type RAS and "cycling RAS mutants," such as KRAS-G12C (Fedele et al., 2018). The effect of KRAS G12C inhibitors is potentiated by coadministration of SHP099, in RASless ( $\mathrm{H}, \mathrm{N}$, and K-RAS null) mouse embryonic fibroblasts reconstituted with KRAS G12C, and in G12Cexpressing cancer cells. Furthermore, co-administration of a MEK inhibitor with SHP099 potentiated tumor shrinkage in MPNST xenografts into sciatic nerve of nude mice. These results suggest promise for treatment of human MPNST with SHP2 inhibitors in combination with other agents blocking RAS-MAPK signal transduction.

Anton Bennett reported on the use of the multiple kinase inhibitor dasatinib in a Ptpn11 mouse model of NS. A phosphoproteomic screen on NS and NSML mouse hearts with cardiomyopathy identified potential drug targets, including a hyper-phosphorylated protein, protein zero-related (PZR) a transmembrane glycoprotein identified as an interacting partner and scaffolding protein for SHP2. NS and NSML-associated mutations promote aberrant SHP2/PZR complexes driving promiscuous downstream signaling. Dasatinib, a multitargeted inhibitor of bcr-abl and Src family kinases approved for pediatric cancers, inhibits PZR hyper-phosphorylation in mice. Low doses of dasatinib in a mouse model of NS improved cardiac function and in NSML prevented progression of HCM.

In a presentation selected from the submitted abstracts, JaeSung Yi reported on using a NSML mouse model to demonstrate the interaction of PZR with SHP2 in the development of HCM. Tyrosyl phosphorylation-defective PZR knock-in mutant mice were generated and intercrossed with NSML mice. Mice expressing tyrosyl phosphorylation-defective PZR alone exhibited normal heart development with normal heart and cardiomyocyte size. Those expressing tyrosyl phosphorylation-defective PZR and NSML were completely protected from the development of $\mathrm{HCM}$ and expressed Anf, Bnp, and Myh7:Myh6 levels comparable to controls. These results support targeting this adapter protein for the treatment of NSML cardiomyopathies.

In a presentation selected from the submitted abstracts, Maja Solman discussed hematopoietic defects in a ptpn11a zebrafish knock-in D61G mutation generated by CRISPR/Cas9. The mutant zebrafish survived to adulthood and displayed growth defects and alterations in craniofacial development. This analysis focused on hematopoiesis during early embryonic development. Mutant embryos had an expansion of hematopoietic stem cells and myeloid lineages including macrophages and neutrophils. Lymphocytes were unaffected. There was a decrease in the number of thrombocytes and erythrocytes. Attenuation of the stem cell expansion occurred using either a MEK inhibitor (Cl1040) or a PI3 Kinase inhibitor (LY294002), indicating that the hematopoietic defects are dependent on the MAP Kinase and PI3 Kinase pathway. Single cell RNA sequencing revealed an expansion of monocyte progenitors with an inflammatory transcriptional signature correctable with dexamethasone.

Rebecca Burdine described a system for screening the pathogenicity of possible RASopathy variants using CRISPR/Cas technology and zebrafish embryos (Jindal et al., 2017). In collaboration with Elizabeth Bhoj, MD, her group identified MAP4K4 as a novel RASopathy gene on patient exome sequencing. MAP4K4 appears to negatively regulate RAS signaling. Thus, loss of function and hypomorphic MAP4K4 alleles mimic the cardiac and craniofacial abnormities caused by increased RAS. This rapid model pipeline should facilitate verification of additional pathogenic RASopathy variants. 
Maria Kontaridis discussed model systems of RASopathy cardiovascular defects. Using human-induced pluripotent stem cell-derived myocytes, she could compare effects of variants in different RASopathy genes (Jaffré et al., 2019). Of note, in Raf1-associated NS mutant cells, while some cellular defects such as incomplete myocyte differentiation and fiber disarray could be rescued by blocking MEK or ERK1/2, cell hypertrophy was rescued by blocking the related kinase ERK5 (Li et al., 2019). These studies suggest drugs to be tested in preclinical models.

Alan Ho described a single-arm Phase II trial of the farnesyl transferase inhibitor tipifarnib in HRAS mutated squamous cell carcinoma (SCC). HRAS mutations occur in a molecular subset of Head and Neck SCC (HNSCC), converting HRAS to an active oncogene. Mutated HRAS is sensitive to tipifarnib, which ultimately prevents HRAS from binding to the membrane, thus rendering it inactive. This trial had two HRAS mutant cohorts: Cohort 1: thyroid cancer; Cohort 2:17 patients with HNSCC. The clinical endpoint was objective response rate. Of the heavily pretreated HNSCC population, six patients had a partial response while four patients had stable disease, one had progressive disease, five were inevaluable, and one was yet to be evaluated. Limiting the analysis to patients with greater than $20 \%$ mutant HRAS allele frequency captured all the study responders and enriched the response rate. An international registration study is currently enrolling to further validate the significant signal observed with tipifarnib in HRAS mutant HNSCC.

An overview of the $\mathrm{NCl}$ Selumetinib in Pediatric Neurofibroma Study (SPRINT) was presented by Andrea Gross. Individuals with neurofibromatosis type 1 (NF1) are prone to develop benign plexiform neurofibromas (PNs), which result from the proliferation of nerve sheath Schwann cells forming nonencapsulated tumors. Currently, surgical resection is the only standard treatment for $\mathrm{PN}$, and this is often not feasible due to the large tumor size and proximity to vital structures. A study performed at the $\mathrm{NCl}$ Clinical Center using the MEK inhibitor selumetinib resulted in unprecedented shrinkage of PNs in NF1 (Dombi et al., 2016). This experience prompted the Clinical Center to initiate the Advancing RASopathies therapies (ART) project, a multicenter approach to developing therapies for non-NF1 RASopathies and RAS driven pediatric tumors. A natural history study will be initiated with the goal of developing meaningful endpoints for future treatment trials. Interventional trials for patients with germline and somatic RAS-driven tumors will be initiated. The integration of natural history and treatment trials will substantially accelerate the understanding of RASopathies, and ultimately lead to the development of effective targeted therapies.

William Timmer presented a brief overview of several National Cancer Institute $(\mathrm{NCl})$ resources: The NCTN Navigator contains tissue and blood samples from adult NCTN Phase III trials (80 trials, 50,000 patients, 600,000 specimens). The NCI Formulary maintains investigational agents. The $\mathrm{NCl}$ Cancer Therapy Evaluation Program maintains a portfolio of investigation drugs available for study its website. Access to these resources requires submission of a letter of intent that will be peer-reviewed. Further information is available on the $\mathrm{NCl}$ website.

\section{4 | Million Dollar Bike Ride 2018 and 2019 grantee reports}

The Penn Medicine Orphan Disease Center organizes an annual fund raising event termed the Million Dollar Bike Ride and provides grants for research on RASopathies. Grantees are invited to present their funded work. Kartik Venkatachalam described the use of Meclizine to attenuate hyperactive RAS-MAP Kinase signaling. RAS isoforms are specific to and dependent upon cholesterol-enriched domains for proper plasma membrane localization. Perturbations disrupting RAS localization attenuate MAP kinase signaling. The recycling of these cholesterol moieties is dependent upon lysosomal exocytosis. Inhibition of lysosomal trafficking reduces plasma membrane cholesterol and inhibits MAP kinase signaling in cells with activated HRAS (Jung et al., 2019; Jung \& Venkatachalam, 2019). The antiemetic, Meclizine, inhibits ethanolamine phosphate cytidylyltransferase 2 (PCYT2), which triggers a signaling cascade that culminates in an increase in sphingomyelin biosynthesis and sequestration of cholesterol. PCYT2 is required for maintenance of mitochondrial iron homeostasis, preventing the translation of sphingolipid biosynthesis enzymes, thus regulating the gain of MAP kinase signaling. Meclizine attenuates MAP kinase signaling and may have use in treatment of hyperactive RAS-MAP kinase-based RASopathies.

Bruce Gelb reported on a Drosophila RASopathy model to screen a large 14,400 compound library of chemicals for potential drug development. Using the Drosophila RAFS257L (a lethal model, 4\% survive to pupae), each chemical was introduced at a variety of concentrations to find rescue from lethality. Four compounds (M1-M4) showed consistent rescue of RAFS257L as well as reduced RAS/ERK activity in vivo. M1 was selected as the lead compound to advance due to its superior chemical structure. Thirty chemical analogs of M1 were developed and the flies treated again; several of these analogs showed improved efficacy. These analogs were further modified and several compounds further increased efficacy. Using a RAF1-induced pluripotent stem cell cardiomyocyte model, one compound normalized cardiomyocyte size. Future work includes testing the most promising compounds in 14 Drosophila RASopathy models with examination of eye and wing phenotype. RAS pathway signaling will be evaluated by western blot analysis to identify compounds with the potential to address multiple RASopathy subtypes.

\section{5 | Clinical trials in RASopathies}

Nancy Ratner moderated a session on treatment endpoints identified in model systems, which could be useful in human trials focusing on the brain and cardiac manifestations of RASopathies. Carlos Prada reviewed mouse models in which a HRAS activating allele (HRASG12V) or NF1 loss of function in mutant oligodendrocytes cause altered myelin structure in the brain and motor dysfunction. Treatment with an antioxidant ( $\mathrm{N}$-acetyl-cysteine) ameliorated phenotypes in these mice (López-Juárez et al., 2017; Mayes et al., 2013; Titus et al., 2017). Based on these results Carlos Prada opened a 
Phase 1 antioxidant clinical trial in children with NF1. Human studies showed that trans-magnetic stimulation and the Physical and Neurological Examination for Soft Signs detect motor system deficits in children with NF1, and these readouts, in combination with MRI results, could be used as endpoints in the NF1 clinical trial.

Tamar Green discussed her ongoing study in children with NF1 or NS (Green, Naylor, \& Davies, 2017; Johnson et al., 2019). Preliminary data show that behavioral features such as attention problems and hyperactivity, as well as atypical behavior, are more common in NS than in NF1. Both groups of children showed frequent attention problems and hyperactivity. Weaknesses in learning and spelling were observed. Measurements of brain region size in NS reveal reduced size of the basal ganglia, which correlated with hyperactivity. Diffusion tensor imaging measurements in NS showed reduced fractional anisotropy indicating possible loss of or aberrant myelin, similar to findings from NF1.

Susan Blaser reviewed neuroimaging findings in RASopathies. She emphasized vascular defects including arteriovenous malformations and fistulas in capillary malformation-arteriovenous malformation syndrome due to RASA1 variants, aneurysms and moya moya as late features of NF1, jugular lymphatic obstruction in NS and persistent occipital and marginal durovenous sinuses in non-NF1 RASopathies. Structural changes in young non-NF1 RASopathy patients included abnormalities in cortical infolding, vertical splenium of the corpus callosum, a small bony posterior fossa with a steeply vertical tentorium and an increase in Chiari 1 malformations. Multifocal white matter ischemic lesions and posterior fossa bleeds were increased in neonates (Cizmeci et al., 2018). Prenatal fetal three-dimensional (3D) ultrasound reconstructions may be helpful in documenting RASopathy facial features at 22-25 weeks gestation (Biard, Steenhaut, Bernard, Race, \& Sznajer, 2019). Imaging features of mosaic RASopathies include brain dysgenesis, intra and extracranial lipomas in encephalo-cutaneous lipomatosis (caused by FGFR1 or KRAS variants) and overlapping features, such as cervical neurofibromas in linear nevus sebaceous syndrome due to a KRAS variant.

\section{6 | Prenatal findings, manifestations, diagnosis, and management}

Pilar Magoulas moderated the session on prenatal manifestations, screening and diagnostic tools for RASopathies. Angie Jelin reviewed prenatal screening for RASopathies by gestational age. In the first trimester (11-14 weeks gestation), measurement of the nuchal translucency (NT) is possible. In the second trimester (18-22 weeks), an anatomy ultrasound can detect major malformations, whereas in the third trimester ( $>24$ weeks gestation), ultrasound is particularly beneficial for monitoring growth and amniotic fluid levels. In a retrospective review of prenatal characteristics identified in fetuses later diagnosed with cardio-facio-cutaneous (CFC) syndrome, 10\% had an abnormal first trimester ultrasound (3/29), 61\% had an abnormal second trimester ultrasound (25/41), and 79\% had an abnormal third trimester ultrasound $(22 / 28)$. The most common first trimester findings included increased NT, cystic hygroma, and abnormal ductus venosus on doppler study. In the second trimester, common abnormal ultrasound findings included polyhydramnios, macrosomia with proportionate short long bones, macrocephaly, skeletal abnormalities, ventriculomegaly, abnormal lymphatic system (thickened nuchal fold, cystic hygroma, hydrops, and ascites), renal abnormalities, and cardiac defects (HCM, pulmonary valve stenosis, septal defects, supraventricular arrhythmia). A 3D ultrasound can show facial features that might otherwise be missed, such as hypertelorism, down-slanting palpebral fissures, long and marked philtrum, low-set posteriorly angulated ears, prefrontal edema, and thick lips. Comparing among RASopathies, fetuses with NS were more likely to show increased NT, distended jugular lymphatic sacs, cystic hygroma, hydrops fetalis, pleural effusion, polyhydramnios, cardiac defects, and renal anomalies. They were less likely to have fetal overgrowth and macrocephaly. Features more common in CFC syndrome include polyhydramnios, renal abnormalities, fetal overgrowth, pleural effusion, and cardiac defects. Fetuses with CS were more likely to show polyhydramnios, fetal overgrowth, and macrocephaly. Prenatal NF1 is rarely diagnosed, however, case reports include polyhydramnios, tumor growth, and macrosomia. Adverse perinatal outcomes including miscarriage, fetal demise and premature delivery occur at a higher frequency.

Sandra Darilek presented prenatal screening and diagnostic testing options. Prenatal suspicion of a RASopathy can be confirmed after CVS or amniocentesis, either by testing for a known familial variant or through RASopathy panel testing. In a study of 845 prenatal cases with RASopathy panel testing consisting of nine genes, 74 variants were found in 72 cases (Leach et al., 2019). Of those, 31 variants were considered pathogenic/likely pathogenic, a large majority in PTPN11 (81\%). There were 43 variants of uncertain significance. The overall diagnostic yield was 3.7\% (31/845; Leach et al., 2019). Of the fetuses with positive results, $64.5 \%$ had a cystic hygroma only, and $20 \%$ had an increased NT only. The highest diagnostic yield based on fetal indication was cystic hygroma with $12 \%(20 / 162)$ having a pathogenic/ likely pathogenic variant. Unpublished data from a single private practice collected over a 4-year period on 14 cases sent for RASopathies panel testing showed a diagnostic yield of $28.6 \%$ (4/14).

Prenatal screening options for RASopathies include two clinically available tests on cell free fetal DNA (cffDNA): PreSeek/Vistara available through Baylor Genetics/Natera and Resura available through Progenity. PreSeek is a single gene cffDNA screening option that assesses 30 genes associated with various conditions and includes screening for 13 RASopathy genes. PreSeek/Vistara screening can be used for singleton pregnancies at $>9$ weeks' gestation, pregnancies with ultrasound finding suggestive of a disorder on the panel, or pregnancies where the father has a confirmed molecular diagnosis of a disorder on the panel. It cannot be used when the mother is affected. In a review of $\sim 1,600$ samples tested using PreSeek, 475 had an indication of abnormal ultrasound or family history. There were 18 cases with positive result for a pathogenic/likely variant in a RASopathy gene. Half were de novo in the fetus, in $22 \%$ (4/18), the variant was identified in the mother and the fetus, and in $28 \%(5 / 18)$, the fetus was positive for a known paternal variant. De novo variants were 
identified in BRAF, HRAS, PTPN11, and RIT1. Indications for screening in positive RASopathy cases included increased NT, cystic hygroma, hydrops, shortened long bones, and father affected with NS.

Resura offers custom cffDNA screen for families with known inherited disorders. This is performed after 10 weeks' gestation and can take 4-8 weeks for test set-up and results. The assay is custom designed for the familial variant. Limited data is available on RASopathy specific cases with validation data reporting 57 cases analyzed for benign variants and fetal genotype was confirmed via Sanger sequencing. Nine cases were performed for known pathogenic variants for autosomal recessive conditions. All fetuses were reportedly unaffected based on Resura and neonatal confirmation testing.

In summary, prenatal testing is available for RASopathies through CVS or amniocentesis. Prenatal screening through cffDNA testing is also available. However, this is not a diagnostic test and requires confirmatory prenatal or postnatal testing. The most common RASopathy genes diagnosed in the prenatal setting include PTPN11, RAF1, KRAS, $B R A F$, and MAP2K1. Genetic counseling is beneficial in assisting families in deciding which option(s) are best for them and to provide anticipatory guidance if a prenatal diagnosis of a RASopathy is confirmed.

\section{7 | Clinical trial endpoints}

The session on clinical trial endpoints was moderated by Richard Klein. Since 1962, under Kefauver-Harris Amendments, the federal drug administration (FDA) requires effectiveness data derived from adequate and well-controlled clinical investigations. Endpoints should demonstrate patient benefit and be accurately measurable using validated instruments, reproducible over time, and across observers. Clinical endpoints demonstrate a direct effect on how patients feel, function, or survive. Clinical outcomes may be represented by increased survival, improvement of cognitive symptoms, or improved quality of life. Alternatively, surrogate endpoints are indirect measurements, such as radiographic images showing reduced tumor size, or blood chemistries. While these are not clinical endpoints in themselves, they may predict a positive clinical outcome. Surrogate endpoints can be used to support accelerated approval, which requires continued study once a medical product is approved demonstrating clinical benefit. Endpoints should also demonstrate cost effectiveness to ensure commercial success and availability through third party payers.

Karin Walsh discussed her study of MEK inhibition on neurocognitive function in children and adults with NF1. She described selecting sensitive and feasible outcome measures for this ancillary cognitive study. Performance-based tests of reaction time, attention, and working memory were administered via a focused computer-based cognitive battery (Cogstate). Real-world executive functions were assessed via parent-rated symptom questionnaire (Behavior Rating Inventory of Executive Function). There was a trend toward improvement in performance-based measures over the first 12 months of treatment, and significant improvement on parent-rated executive functions observable at the 6-month follow-up and increasing by 12 months. Analyzing the possible impact of changes in pain and physical functioning is important in fully interpreting these results and additional research is necessary given the lack of a comparison group.

Pamela Wolters talked about the use of patient reported outcomes (PROs) in descriptive and natural history studies as well as clinical trials. PROs (reported by the patients themselves) differ from clinician-reported outcomes (collected by a clinical investigator, physician, or other health provider) and observer-reported outcomes (collected by caretakers; Acquadro et al., 2003). General quality of life (QOL) scales evaluate a wide range of domains, including physical, social, and emotional functioning. Disease-specific QOL scales focus on specific functions affected by a specific medical condition, and symptom-specific tools assess one symptom, such as pain, in any medical condition (Luckett \& King, 2010). PRO measures may provide data on positive outcomes, like improvements in functioning, as well as negative effects, such as treatment-related toxicities. One main challenge in the use of PRO measures in clinical trials for RASopathies is that less self-reporting is achievable with young children and individuals with cognitive disabilities. Approaches that can improve selfreported data collection for children with cognitive impairment include use of pictures, simplified language, more white space around text, and having information/questions read aloud (Kramer \& Schwartz, 2017). The use of wearables may elucidate otherwise difficult to assess data (Slade et al., 2018). For example, increased physical activity might be used as a measure of reduced pain.

No validated PRO measures specific to RASopathies exist. Researchers need to evaluate PRO measurement scales to determine which are appropriate for RASopathy trials and prioritize tools necessary to fill in data gaps. Benefits of getting PROs approved through the FDA Clinical Outcome Assessment Qualification Program may include faster review time, more meaningful outcomes, and quicker availability of new therapeutic approaches (U.S. Food and Drug Administration, 2009). However, the FDA's program for development and validation of PROs is a long, rigorous process.

Annie Kennedy from parent project muscular dystrophy (PPMD) talked about working with industry, families, patients, and Certified Duchenne Care Centers to centralize data in the Duchenne Outcomes Research Interchange. Different registries collected valuable, but siloed data. Basic tools, such as how to measure walking ability, vary among researchers, leading to inconsistent and problematic results. PPMD brings data sources together and validates the data. The Duchenne Regulatory Science Consortium was formed under the Critical Path Institute to develop tools and optimize trial designs. While tighter inclusion criteria can lead to faster results and earlier approval, payers may restrict access based on results in a limited study population. PPMD and other advocacy organizations were originally focused on regulatory approval, rather than data necessary to inform payers, which can result in restricted access. While patients, industry, and regulators all prioritized biomarkers as important, payers did not. Payers are recognizing caregiver burdens and the effects on entire families with higher costs. Thus, QOL measures might provide evidence of overall lower cost of care. PPMD is leading efforts to evaluate the 
prioritization of outcomes through a broad range of stakeholders including patients, parents, methodologists, industry, physicians, clinicians, and payers.

\section{8 | Advocacy groups panel on clinical trial endpoints}

A discussion was moderated by Michelle Ellis, an adult advocate with NS. The panel consisted of a representative from each advocacy group: Tuesdi Dyer from CFC International; Angelica Thomas from Costello Syndrome Family Network; Alwyn Dias from Children's Tumor Foundation; Gregg Erickson from Neurofibromatosis Network; and Amanda Brown from Noonan Syndrome Foundation. Patient advocates highlighted pain, PNs, intractable seizures, neurocognitive function, and social skills as the most urgent treatment targets. Further studies on how RASopathies impact the lives of adults were requested. The heterogeneity of RASopathies, not only on the genetic level but also among individuals affected by the same syndromic condition makes identification of meaningful endpoints challenging. The audience was reminded that affected individuals are keen to participate in research studies. Adults deciding about study participation were willing to accept a higher risk for themselves, in contrast to what they would accept for their child. Difficulties can arise when a treatment trial ends and the study drug is no longer available to participants.

\section{2 | CONCLUSION}

In conclusion, significant therapeutic effects of pathway specific medications on life altering complications of RASopathies have already been reported, such as the use of selumetinib for plexiform neurofibromata in NF1 (Dombi et al., 2016) or, most recently, trametinib for HCM in NS (Andelfinger et al., 2019). Ongoing research on drug development and delivery as discussed during this meeting promises further progress toward precision medicine for RASopathies and associated medical complications. Patients, clinician scientists, laboratory scientists, drug companies, and regulatory bodies are coming together to promote such outcomes driven research.

\section{ACKNOWLEDGMENTS}

In addition to the NIH (1R13TR002780-01), the meeting was financially supported by an anonymous donor; the National Cancer Institute; IGIA pharmaceuticals; KURA oncology; Boehringer Ingelheim; Prevention Genetics; UAB School of Medicine; Children's Tumor Foundation; and International Costello Syndrome Support Group. Linda de Vries and David Chitayat contributed to planning the presentation given by Susan Blaser.

\section{CONFLICT OF INTEREST}

The authors do not declare a conflict of interest in regard to this manuscript.

\section{DATA AVAILABILITY STATEMENT}

$\mathrm{N} / \mathrm{A}$

\section{ORCID}

Karen W. Gripp (D) https://orcid.org/0000-0001-8200-1733

Katherine A. Rauen (D) https://orcid.org/0000-0003-1719-7228

Martin Zenker (D) https://orcid.org/0000-0003-1618-9269

\section{REFERENCES}

Acquadro, C., Berzon, R., Dubois, D., Leidy, N. K., Marquis, P., Revicki, D., ... PRO Harmonization Group. (2003). Incorporating the patient's perspective into drug development and communication: An ad hoc task force report of the patient-reported outcomes (PRO) harmonization group meeting at the Food and Drug Administration. Value in Health, 6, 522-531. https://doi.org/10.1046/j.1524-4733.2003.65309.x

Andelfinger, G., Marquis, C., Raboisson, M. J., Théoret, Y., Waldmüller, S., Wiegand, G., ... Hofbeck, M. (2019). Hypertrophic cardiomyopathy in Noonan syndrome treated by MEK-inhibition. Journal of the American College of Cardiology, 73, 2237-2239. https://doi.org/10.1016/j.jacc. 2019.01.066

Araki, T., Mohi, M. G., Ismat, F. A., Bronson, R. T., Williams, I. R., Kutok, J. L., ... Neel, B. G. (2004). Mouse model of Noonan syndrome reveals cell type- and gene dosage-dependent effects of Ptpn11 mutation. Nature Medicine, 10, 849-857. https://doi.org/10.1038/nm1084

Biard, J. M., Steenhaut, P., Bernard, P., Race, V., \& Sznajer, Y. (2019). Antenatal diagnosis of cardio-facio-cutaneous syndrome: Prenatal characteristics and contribution of fetal facial dysmorphic signs in utero. European Journal of Obstetrics \& Gynecology, 240, 232-241. https:// doi.org/10.1016/j.ejogrb.2019.06.035

Castel, P., Cheng, A., Cuevas-Navarro, A., Everman, D. B., Papageorge, A. G., Simanshu, D. K., ... McCormick, F. (2019). RIT1 oncoproteins escape LZTR1-mediated proteolysis. Science, 363, 1226-1230. https://doi.org/10.1126/science.aav1444

Cizmeci, M. N., Lequin, M., Lichtenbelt, K. D., Chitayat, D., Kannu, P., James, A. G., ... de Vries, L. S. (2018). Characteristic MR imaging findings of the neonatal brain in RASopathies. American Journal of Neuroradiology, 39, 1146-1152. https://doi.org/10.3174/ajnr.A5611

Dombi, E., Baldwin, A., Marcus, L. J., Fisher, M. J., Weiss, B., Kim, A., ... Widemann, B. C. (2016). Activity of Selumetinib in Neurofibromatosis type 1-related plexiform neurofibromas. The New England Journal of Medicine, 375, 2550-2560. https://doi.org/10.1056/NEJMoa1605943

Fedele, C., Ran, H., Diskin, B., Wei, W., Jen, J., Geer, M. J., ... Tang, K. H. (2018). SHP2 inhibition prevents adaptive resistance to MEK inhibitors in multiple cancer models. Cancer Discovery, 8, 1237-1249. https:// doi.org/10.1158/2159-8290.CD-18-0444

Green, T., Naylor, P. E., \& Davies, W. (2017). Attention deficit hyperactivity disorder (ADHD) in phenotypically similar neurogenetic conditions: Turner syndrome and the RASopathies. Journal of Neurodevelopmental Disorders, 9, 25.

Gripp, K. W., Aldinger, K. A., Bennett, J. T., Baker, L., Tusi, J., PowellHamilton, N., ... Dobyns, W. B. (2016). A novel rasopathy caused by recurrent de novo missense mutations in PPP1CB closely resembles Noonan syndrome with loose anagen hair. American Journal of Medical Genetics. Part A, 170, 2237-2247. https://doi.org/10.1002/ajmg.a.37781

Jaffré, F., Miller, C. L., Schänzer, A., Evans, T., Roberts, A. E., Hahn, A., \& Kontaridis, M. I. (2019). Inducible pluripotent stem cell-derived cardiomyocytes reveal aberrant extracellular regulated kinase 5 and mitogen-activated protein kinase kinase $1 / 2$ signaling concomitantly promote hypertrophic cardiomyopathy in RAF1-associated Noonan syndrome. Circulation, 140, 207-224. https://doi.org/10.1161/ CIRCULATIONAHA.118.037227

Jindal, G. A., Goyal, Y., Yamaya, K., Futran, A. S., Kountouridis, I., Balgobin, C. A., ... Shvartsman, S. Y. (2017). In vivo severity ranking of 
Ras pathway mutations associated with developmental disorders. Proceedings of the National Academy of Sciences of the United States of America, 114, 510-515. https://doi.org/10.1073/pnas.1615651114

Johnson, E. M., Ishak, A. D., Naylor, P. E., Stevenson, D. A., Reiss, A. L., \& Green, T. (2019). PTPN11 gain-of-function mutations affect the developing human brain, memory, and attention. Cerebral Cortex, 29, 2915-2923. https://doi.org/10.1093/cercor/bhy158

Jung, J., Cho, K. J., Naji, A. K., Clemons, K. N., Wong, C. O., Villanueva, M., ... Venkatachalam, K. (2019). HRAS-driven cancer cells are vulnerable to TRPML1 inhibition. EMBO Reports, 20, e46685. https://doi.org/10. 15252/embr.201846685

Jung, J., \& Venkatachalam, K. (2019). TRPML1 and RAS-driven cancers: Exploring a link with great therapeutic potential. Channels (Austin, Tex.), 13, 374-381. https://doi.org/10.1080/19336950.2019.1666457

Koczkowska, M., Chen, Y., Callens, T., Gomes, A., Sharp, A., Johnson, S., ... Messiaen, L. M. (2018). Genotype-phenotype correlation in NF1: Evidence for a more severe phenotype associated with missense mutations affecting NF1 codons 844-848. American Journal of Human Genetics, 102, 69-87. https://doi.org/10.1016/j.ajhg.2017.12.001

Kramer, J. M., \& Schwartz, A. (2017). Reducing patient barriers to patientreported outcome measures for people with cognitive impairments. Archives of Physical Medicine and Rehabilitation, 98, 1705-1715. https://doi.org/10.1016/j.apmr.2017.03.011

Leach, N. T., Wilson Matthews, D. R., Rosenblum, L. S., Zhou, Z., Zhu, H., \& Heim, R. (2019). Comparative assessment of gene-specific variant distribution in prenatal and postnatal cohorts tested for Noonan syndrome and related condition. Genetics in Medicine, 21, 417-425. https://doi.org/10.1038/s41436-018-0062-0

Li, R., Baskfield, A., Lin, Y., Beers, J., Zou, J., Liu, C., ... Zheng, W. (2019). Generation of an induced pluripotent stem cell line (TRNDi003-A) from a Noonan syndrome with multiple lentigines (NSML) patient carrying a p.Q510P mutation in the PTPN11 gene. Stem Cell Research, 34, 101374. https://doi.org/10.1016/j.scr.2018.101374

López-Juárez, A., Titus, H. E., Silbak, S. H., Pressler, J. W., Rizvi, T. A., Bogard, M., ... Ratner, N. (2017). Oligodendrocyte Nf1 controls aberrant notch activation and regulates myelin structure and behavior. Cell Reports, 19, 545-557. https://doi.org/10.1016/j.celrep.2017.03.073

Luckett, T., \& King, M. T. (2010). Choosing patient-reported outcome measures for cancer clinical research-Practical principles and an algorithm to assist non-specialist researchers. European Journal of Cancer, 46, 3149-3157. https://doi.org/10.1016/j.ejca.2010.08.002

Ma, L., Bayram, Y., McLaughlin, H. M., Cho, M. T., Krokosky, A., Turner, C. E., ... Chung, W. K. (2016). De novo missense variants in PPP1CB are associated with intellectual disability and congenital heart disease. Human Genetics, 135, 1399-1409.

Mayes, D. A., Rizvi, T. A., Titus-Mitchell, H., Oberst, R., Ciraolo, G. M., Vorhees, C. V., ... Ratner, N. (2013). Nf1 loss and Ras hyperactivation in oligodendrocytes induce NOS-driven defects in myelin and vasculature. Cell Reports, 26, 1197-1212. https://doi.org/10.1016/j.celrep. 2013.08.011

Oba, D., Inoue, S. I., Miyagawa-Tomita, S., Nakashima, Y., Niihori, T., Yamaguchi, S., ... Aoki, Y. (2018). Mice with an oncogenic HRAS mutation are resistant to high-fat diet-induced obesity and exhibit impaired hepatic energy homeostasis. eBioMedicine, 27, 138-150. https://doi.org/10.1016/j.ebiom.2017.11.029

Schuhmacher, A. J., Guerra, C., Sauzeau, V., Cañamero, M., Bustelo, X. R., \& Barbacid, M. (2008). A mouse model for Costello syndrome reveals an Ang II-mediated hypertensive condition. The Journal of Clinical Investigation, 118, 2169-2179. https://doi.org/10.1172/ $\mathrm{JCl} 34385$

Slade, A., Isa, F., Kyte, D., Pankhurst, T., Kerecuk, L., Ferguson, J., ... Calvert, M. (2018). Patient-reported outcome measures in rare diseases: A narrative review. Orphanet Journal of Rare Diseases, 13, 61. https://doi.org/10.1186/s13023-018-0810-x

Tidyman, W. E., \& Rauen, K. A. (2016). Pathogenetics of the RASopathies. Human Molecular Genetics, 25, R213-R132. https://doi.org/10.1093/ hmg/ddw191

Titus, H. E., López-Juárez, A., Silbak, S. H., Rizvi, T. A., Bogard, M., \& Ratner, N. (2017). Oligodendrocyte RasG12V expressed in its endogenous locus disrupts myelin structure through increased MAPK, nitric oxide, and notch signaling. Glia, 65, 1990-2002. https://doi.org/10. 1002/glia.23209

U.S. Food and Drug Administration. Guidance for industry. Patientreported outcome measures: use in medical product development to support labeling claims. 2009. Retrieved from https://www.fda.gov/ regulatory-information/search-fda-guidance-documents/patientreported-outcome-measures-use-medical-product-developmentsupport-labeling-claims

Urosevic, J., Sauzeau, V., Soto-Montenegro, M. L., Reig, S., Desco, M., Wright, E. M., ... Barbacid, M. (2011). Constitutive activation of B-Raf in the mouse germ line provides a model for human cardio-faciocutaneous syndrome. Proceedings of the National Academy of Sciences of the United States of America, 108, 5015-5020. https://doi.org/10. 1073/pnas.1016933108

Yao, Z., Gao, Y., Su, W., Yaeger, R., Tao, J., Na, N., ... Rosen, N. (2019). RAF inhibitor PLX8394 selectively disrupts BRAF dimers and RASindependent BRAF-mutant-driven signaling. Nature Medicine, 25, 284-291. https://doi.org/10.1038/s41591-018-0274-5

Young, L. C., Hartig, N., Boned Del Río, I., Sari, S., Ringham-Terry, B., Wainwright, J. R., ... Rodriguez-Viciana, P. (2018). SHOC2-MRAS-PP1 complex positively regulates RAF activity and contributes to Noonan syndrome pathogenesis. Proceedings of the National Academy of Sciences of the United States of America, 115, E10576-E10585. https:// doi.org/10.1073/pnas.1720352115

How to cite this article: Gripp KW, Schill L, Schoyer L, et al. The sixth international RASopathies symposium: Precision medicine-From promise to practice. Am J Med Genet Part A. 2019;1-10. https://doi.org/10.1002/ajmg.a.61434 